\title{
Clinico-Aetiological Scenario and Microbiological Profile in Corneal Ulcer Cases in a Tertiary Eye Care Centre in Southern Assam
}

\author{
Nilanjan Kaushik Thakurr ${ }^{1}$ Atanu Chakravarty², Shibashis Deb ${ }^{3}$, Subhashish Deb4 ${ }^{4}$ Ankita Narula ${ }^{5}$ \\ ${ }^{1}$ Department of Ophthalmology, Silchar Medical College, Silchar, Assam, India. ${ }^{2}$ Department of \\ Microbiology, Jorhat Medical College, Jorhat, Assam, India. ${ }^{3}$ Department of Ophthalmology, Silchar \\ Medical College, Silchar, Assam, India. ${ }^{4}$ Department of Ophthalmology, Silchar Medical College, \\ Silchar, Assam, India. ${ }^{5}$ Department of Ophthalmology, PGIMS, Rohtak, Haryana, India.
}

\section{ABSTRACT}

\section{BACKGROUND}

Corneal ulcer is a global burden which is an ocular emergency. The outcome of corneal ulcer depends on its management. Aetiology, microbiological flora and antibiotic sensitivity vary from region to region. We wanted to analyse the epidemiology of corneal ulcer in Barak valley region of Assam.

\section{METHODS}

This is a cross-sectional study conducted from May 2017 to June 2018 at Silchar Medical College, Silchar, Assam, India. Cases of corneal ulcer in the study period were included. Corneal scrapping was done, and the sample was sent for Gram stain, $\mathrm{KOH}$ mount stain and culture-sensitivity testing.

\section{RESULTS}

A total of 122 patients were examined and 70 cases had positive microbiological findings. Pure bacterial isolates were found in 37 (52.8\%) of the 70 cultures and pure fungal isolates in $23(32.8 \%)$ of the cultures. Staphylococcus aureus was the most commonly isolated bacterial organism (42.5\%). Out of fungal isolate, Aspergillus spp. (46.7\%), was the commonest. Farmers were the most commonly affected work group.

\section{CONCLUSIONS}

Although the major aetiological agents in our study were bacterial, fungal ulcers were also seen in a significant proportion of patients. Staphylococcus was the major bacterial isolate and Aspergillus was the major fungal isolate.

\section{KEY WORDS}

Corneal Ulcer, North-East India, Staphylococcus, Aspergillus
Corresponding Author: Dr. Shibashis Deb, S/o. Dr. Dipankar Deb, New P. G. Hostel, Room No. 110, Silchar Medical College, Silchar, Cachar-788014, Assam, India.

E-mail:debshibashis@gmail.com

DOI: $10.14260 / \mathrm{jemds} / 2020 / 164$

Financial or Other Competing Interests: None.

How to Cite This Article:

Thakur NK, Chakravarty A, Deb S, et al. Clinico-aetiological scenario and microbiological profile in corneal ulcer cases in a tertiary eye care centre in Southern Assam. J. Evolution Med. Dent. Sci. 2020;9(10):753-758, DOI: $10.14260 /$ jemds/2020/164

Submission 27-11-2019, Peer Review 06-02-2020,

Acceptance 14-02-2020, Published 09-03-2020. 


\section{BACKGROUND}

Corneal ulcer is a sight threatening, monocular disorder affecting a significant percentage of the world population irrespective of age or gender. In various studies, it has been found that corneal diseases are the second-most leading cause of blindness world-wide, of which corneal ulcer is a significant contributor. ${ }^{1}$ Blindness due to corneal ulcer affects more in developing and third-world countries ${ }^{2}$ and thus study of its etiological scenario in southern Assam is of special interest. Cornea is a unique structure by its structural and functional identity. Being situated in the most vulnerable exposed portion of the eyeball, it undergoes repeated microinjuries and remain in constant exposure to microbiological agents. Various micro-organisms are responsible for corneal ulcer of which some are commensal normally present in the conjunctiva other present in the surrounding environment. In most of the cases of corneal ulcers prompt treatment results in successful healing of ulcer but sometimes these may fail to respond and are labelled as non-healing corneal ulcers.

The outcome of corneal ulcer is myriad depending on its management. It may resolve without any sequelae, it may perforate leading to devastating consequences or it may heal but lead to opacification which, if located centrally, cause loss of vision. The literature is rife with exhaustive list of various risk factors for corneal ulcers and its myriad modes of presentation. But there is a wide regional variation to it, depending on the climate, culture, socioeconomic status, common occupation, habits to name a few factors. The antibiotic sensitivity of the pathogenic organism also differs in various regions. For this reason, the global data doesn't necessarily correlate with the local data. Considering the impact of corneal ulcer as an important preventable causative factor for monocular visual loss world-wide, there is a surprising dearth of studies done in the north-east India to evaluate the etiological factors of corneal ulcer. ${ }^{3}$ In India, an epidemiological population based study on aetiology of corneal ulcer was conducted in Madurai, ${ }^{4}$ which estimates that the incidence of corneal ulcer is 10 times higher than age and sex-adjusted American population ${ }^{5}$. Thus, a study on aetiology of corneal ulcer is the need of the hour in north-east India.

\section{Aims and Objectives}

1. Study the epidemiology of corneal ulcer in Barak valley region of Assam over 1 year period from May 2017 to June 2018.

2. Evaluate the age and sex predilections, occupational hazards and various risk factors.

3. Evaluate the causative organisms of corneal ulcer to adopt a systematic, evidence-based protocol in preventing and treating corneal ulcer.

\section{METHODS}

It is a cross-sectional study was done from May 2017 to June 2018 conducted at Department of Ophthalmology, Silchar Medical College. A total of 122 patients with corneal ulcer which is defined as loss of corneal epithelium with underlying infiltration and suppuration with surrounding inflammation with/without hypopyon, presenting in our hospital in the time frame of May 2017 to June 2018 were included in the study. The sample size was taken based on the convenience of the study. The catchment area of our hospital has population of 36 lakh, covering three districts-Cachar, Hailakandi and Karimganj. Most of the patients come from mid- to lowsocioeconomic strata, which has been found out by modified Kuppuswamy scale and have agriculture-based occupation. Almost all cases were advised admission. In all these patients, the presenting symptoms, ulcer duration, history of systemic conditions like diabetes, immunosuppression and prolonged hospitalization and trauma, if any, which is relevant to corneal ulcer were asked and recorded. Those patients with associated viral keratitis or ulcers due to chemical injury were excluded from the study. The study was approved by Institute of Ethical Committee and informed consent was obtained.

\section{Clinical Procedures}

The visual acuity was measured in all the cases and a detailed anterior segment examination with a slit lamp biomicroscope was performed. The ulcer was examined under the following criteria- size, location, number, depth and extent of infiltrations, satellite ulcers, margin and base of ulcer, presence/absence of hypopyon, scleral extension and corneal thinning. Any local eye conditions which are known to predispose to formation of corneal ulceration like chronic dacryocystitis, exposure keratitis, trichiasis, history of trauma with vegetative materials and pre-existing corneal opacity/degeneration were noted. Proper documentation and colour coding of every case was also done. After the clinical diagnosis was made, our next objective was to identify the microbiological profile of the ulcer. For obtaining the sample, first the affected eye was anaesthetised topically with $0.5 \%$ proparacaine eye drop. Samples were obtained by scrapping with a sterile 26 gauze needle from the ulcer base and edges. The sample was then inoculated into - a) gram-stain slide b) $\mathrm{KOH}$ mount slide and by linear streaking in c) blood media agar and d) Sabouraud Dextrose agar and sent for microbiological examination. The major occupation in our region is farming which has been reported to be associated with higher incidence of fungal corneal ulcers, which prompted us to send for $\mathrm{KOH}$ smear and SDA agar before starting any empirical antibiotics. After sending the sample, the patients were started on empirical antibiotics - $0.5 \%$ Moxifloxacin eye drop, 1\% Atropine eye drop, oral NSAIDs, PPI and Vitamin C tablets. Bacterial cultures were reported after $48 \mathrm{hrs}$ of incubation. In cases of gram positive cocci, the same treatment was continued. If gram negative cocci/bacilli was reported, $0.3 \%$ tobramycin eye drop was added. Fortified Cefotaxime and Amikacin eye drops were used in cases with positive sensitivity reports. The majority of the population in our region are associated with agriculture with poor literacy rate, generally they gave unreliable history, and our protocol was to initiate antifungal eye drop if there is clinical suspicion of fungal corneal ulcer. 5\% Natamycin eye drop was used as first line treatment for fungal ulcers. Topical Itraconazole was added in cases of resistant ulcers. Progress of the ulcer was noted every day, by measuring the size of the ulcer, hypopyon if present, reduction in infiltrations, improvement in the symptoms of the patient and proper documentation was 
done. Usually when the ulcer started to regress and there was good patient compliance, they were discharged. Routine weekly follow up was done.

\section{Laboratory}

Bacterial growth in two solid phase media or confluent growth in one media was considered significant. Observation of fungal hyphal filaments in $\mathrm{KOH}$ smear or isolation of fungal growth in SDA (Sabouraud's Dextrose Agar) was considered sufficient for diagnosis.

\section{Statistical Analysis}

The Statistical Package for Social Science \{SPSS Version 20 will be used for Data Analysis. Mean, median, and SD are used to describe quantitative data. Qualitative data are summarized using frequency and percentage.

\section{RESULTS}

\section{Epidemiological Characteristics}

The study takes into account the total number of patients attending our department in the allotted time period, and compares the total number of corneal ulcers cases of infective aetiology out of them. All 122 patients met the criteria for the clinical diagnosis: a defect of the corneal epithelium, associated with underlying stromal infiltration, suppuration and inflammation. The mid-period study population served by our hospital was 36 lakh yielding an incidence of 3.38 per 100,000 person-years. Of the total 122 patients $87(71.3 \%)$ were males and 35 (28.7\%) were females. Incidence of corneal ulceration occurred most frequently in the middle decades of life, in both the groups. However it was observed that corneal ulcer occurred more frequently in males then in females in the middle decades in the ratio 2.5 to 1 .

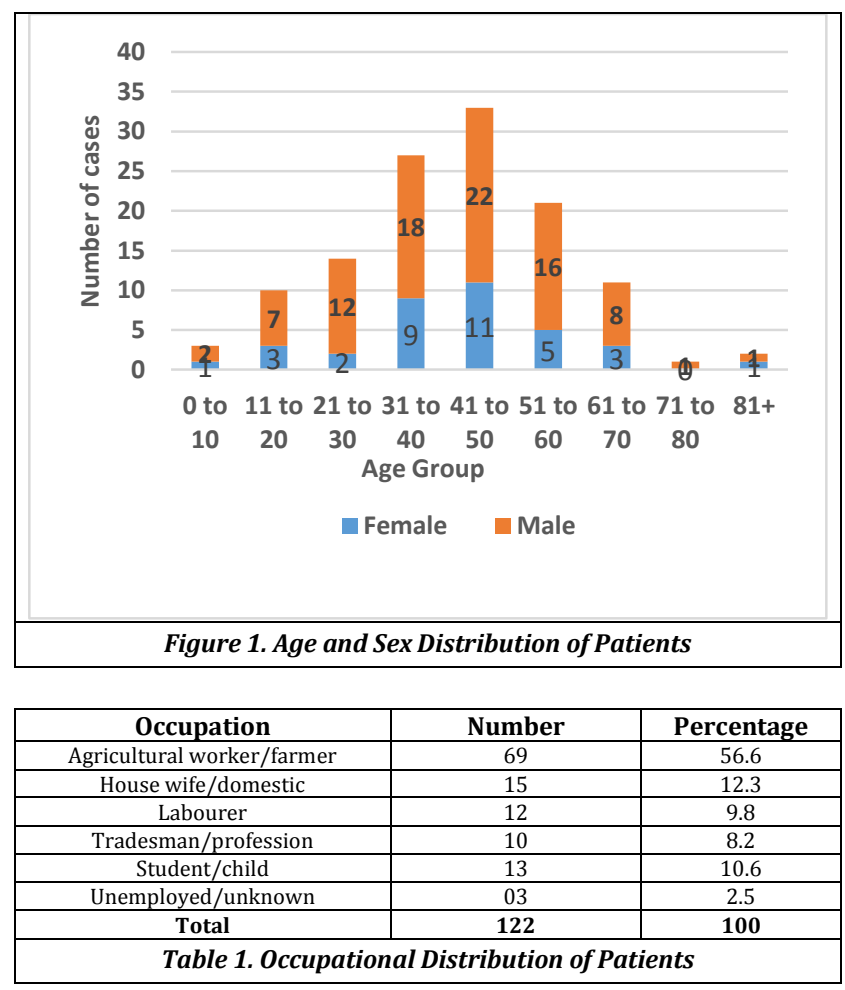

The occupations of the patients reflected a cross-section of the work force in Barak valley and the surrounding area. Majority of the population was engaged in agriculture and animal husbandry. The occupation of the population has been shown in Table 1. The duration of illness of the patients before they presented at Silchar Medical College and Hospital was also determined. It was noted that, most of the patients presented within 14 days after the onset of their illness. Majority of the patients (68 patients, 55.7\%) were seen from day 10 to 14 , but it was noted that, 14 patients waited for more than a month before evaluation. Before presenting at Silchar Medical College and Hospital, 86 patients (70.5\%) of the total 122 were treated. Of the 86 patients who received medical attention 40 patients (32.8\%) were seen by ophthalmologists, 09 patients $(7.3 \%)$ went to general healthcare professionals, 37 patients $(30.3 \%)$ received advice from chemists, pharmacists, homeopaths or health assistants. It was noted that, in several instances patients consulted both general health workers and quacks before presenting to an ophthalmologist. Remaining 36 patients (29.5\%) did not seek any prior medical help.

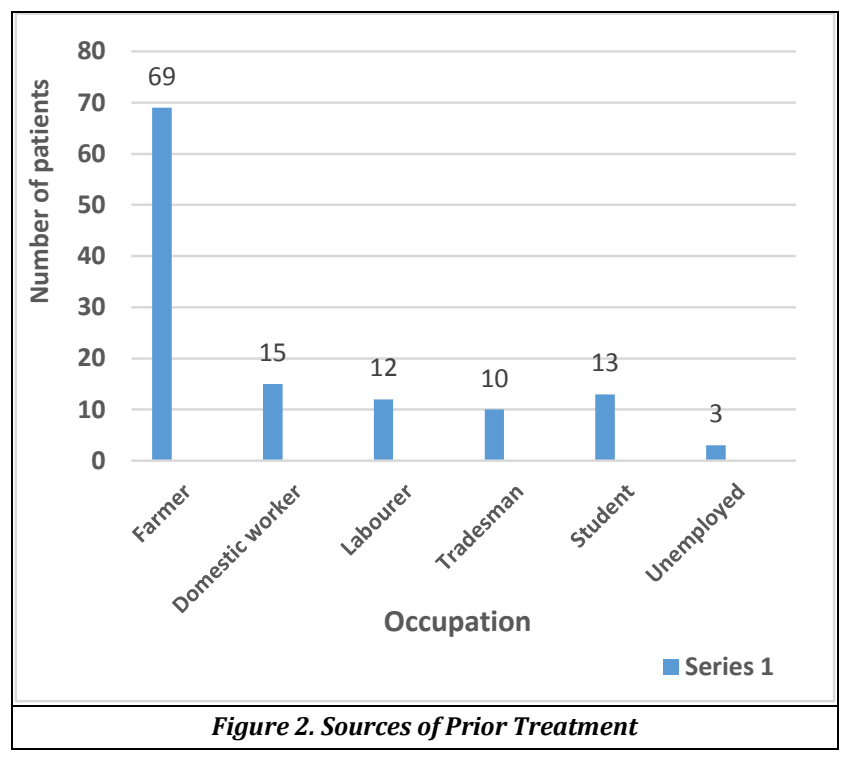

Of the 86 patients who presented on some form of topical medication, 51 patients $(59.3 \%)$ were taking topical antibiotics, usually moxifloxacin, ciprofloxacin, gentamicin or chloramphenicol; 8 patients $(9.3 \%)$ were taking antifungal drops, usually natamycin; 17 patients $(19.7 \%)$ were taking topical corticosteroids; and 10 (11.6\%) were taking unknown drops. Depending on the severity of the ulcer, widespread use of topical antibiotics, antifungals, and corticosteroids was noted, either alone or in combination. Patients who visited ophthalmologists or general physicians prior to reporting in our institute were using antibiotic eye drops, especially moxifloxacin or ciprofloxacin, while use of chloramphenicol, gentamicin, topical corticosteroids were seen to be in cases who visited pharmacists, homeopaths, chemists or quacks. Use of unknown or herbal products were also found exclusively in this group of patients. 


\section{Risk Factors}

The patients were evaluated to found out any prevalent risk factor in our region. A history of recent corneal trauma with was obtained in $41(33.6 \%)$ of the 122 patients. In most of the patients the causative agent was agricultural or animal products, though in a small number of patients, they could not specify the causative agent. Conditions which predispose or aggravate corneal ulceration were present in 21 (17.2\%) of the 122 patients. These risk factors included chronic dacryocystitis in 7 patients, 9 patients had uncontrolled diabetes, 2 patients presented with entropion with trichiasis and 3 patients had exposure keratitis due to Bell's palsy.

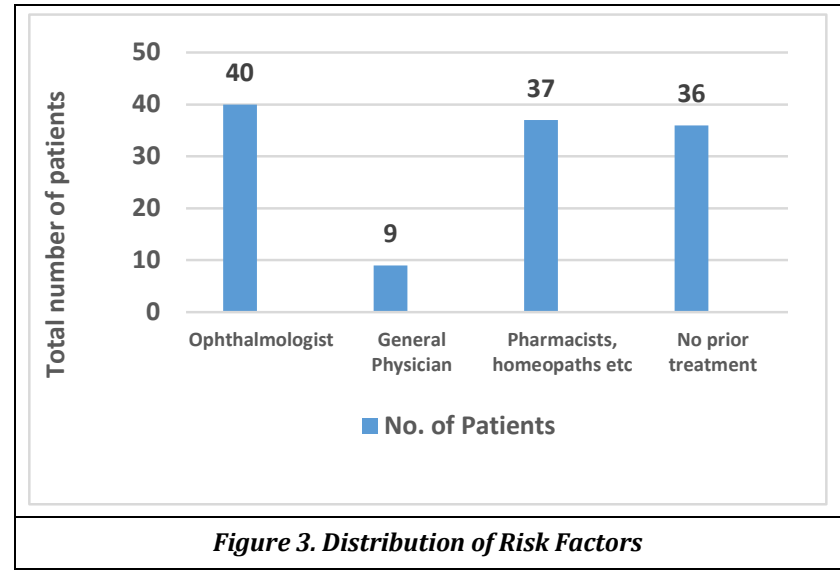

\section{Microbiological Diagnosis}

Of the 122 patients in whom the scrapping sample was sent, in $70(57.37 \%)$ cases, the microbiological report was positive and they fulfilled the required criteria. Single bacterial isolate was present in 37 (52.8\%) of the 70 cultures performed and single fungal isolate in $23(32.8 \%)$ of the cultures. In some cases, mixed microbial isolate was present - in a total of 10 $(14.3 \%)$ out of 70 positive cultures. Of the 40 isolates, 28 (73.3\%) were Gram positive and 12 (26.6\%) were Gram negative. Staphylococcus aureus was the most commonly isolated bacterial organism representing 17 (42.5\%) of all positive bacterial cultures. The next most commonly isolated Gram-positive organism was Streptococcus pneumoniae with $6(15 \%)$ positive cultures. Staphylococcus epidermidis was cultured from 4 patients (10\%). Of the 12 gram-negative isolates, $6(15 \%)$ isolates of Pseudomonas aeruginosa were isolated. $4(10 \%)$ were of Neisseria gonorrhoeae. Rest of the cultures showed either mixed growth, or specific organism could not be isolated.

\begin{tabular}{|c|c|c|}
\hline Bacterial Isolate & Total No. of Isolates & Percentage \\
\hline Gram Positive isolates & $\mathbf{2 8}$ & $\mathbf{7 3 . 3 \%}$ \\
\hline Staphylococcus aureus & 17 & $42.5 \%$ \\
\hline Streptococcus pneumoniae & 06 & $15 \%$ \\
\hline Staphylococcus epidermidis & 04 & $10 \%$ \\
\hline Gram Negative isolates & $\mathbf{1 2}$ & $\mathbf{2 6 . 6 \%}$ \\
\hline Pseudomonas aeruginosa & 06 & $15 \%$ \\
\hline Neisseria gonorrhoeae & 04 & $10 \%$ \\
\hline \multicolumn{3}{|c|}{ Table 2. Bacterial Isolates } \\
\hline
\end{tabular}

A total of 30 fungal isolates were cultured from 70 positive corneal ulcers -23 pure isolates and 7 mixed isolate. Of 30 total isolates, 14 were Aspergillus spp. (46.7\%), 6 were Fusarium spp. (20\%) and 3 were Candida spp. (10\%). The rest were mixed isolates.

\begin{tabular}{|c|c|c|}
\hline Fungal Isolate & Total No. of Isolates & Percentage \\
\hline Aspergillus spp. & 14 & $46.7 \%$ \\
\hline Fusarium spp. & 06 & $20 \%$ \\
\hline Candida spp. & 03 & $10 \%$ \\
\hline \multicolumn{3}{|c|}{ Table 3. Fungal Isolates } \\
\hline
\end{tabular}

\section{DISCUSSION}

The prevalence of visual disability from corneal opacity invariably varies from one geographical location to another, as per the environmental condition, prevalent occupation of the populace, socio-economic condition of the population and in areas in the world where trachoma and other infectious causes of eye disease are endemic, blindness rates may be as high as $3 \%$ of the entire population. Various surveys in Africa and Asia and worldwide have consistently listed corneal scarring as a result of corneal ulcer to be a leading cause of visual impairment or blindness, second only to cataract.6-9

In southern Assam, reliable statistical demographic data documenting the prevalence of blindness from corneal scarring are not available, but the mid-period study population served by our hospital was 36 lakhs yielding an incidence of 3.38 per 100,000 person-years. In our study we found that, the males in their fourth decade (14.7\%) and fifth decade (18\%) of life are particularly at risk and the ratio of male to female patients was 2.5 to 1 , whereas Gonzales et al, in their study on the population of Madurai district, reported a ratio of 1.5 to 1.4 The high incidence of corneal ulcer in the middle age group ( $4^{\text {th }} \& 5^{\text {th }}$ decade), in both sexes, is possibly due to being more physically active and thus more predisposed to exposure to risk factors.

In our study, it was found that the occupation of majority of ulcer patients was related to agriculture and animal husbandry (69\%), which is similar to occupational profile of Nepal $(72 \%),{ }^{10}$ but markedly contrasted with that of Ghana, where only $(16.1 \%)$ of the patients were involved in agricultural activity. ${ }^{11}$ Most of the patients who were associated with agriculture were either paddy field workers or tea garden labourers. Medical help was sought by 86 $(70.5 \%)$ of the 122 patients before their initial examination at Silchar Medical College and Hospital. Of the 86 patients who received medical attention 40 patients $(32.8 \%)$ were seen by eye physicians, 09 patients $(7.3 \%)$ went to general physicians, 37 patients $(30.3 \%)$ received advice from chemists, pharmacists, homeopaths or health assistants. In several instances patients consulted both physicians and village healers before presenting for examination. Remaining 36 patients $(29.5 \%)$ did not seek any prior medical help.

It is has been found that majority of the patients (55.7\%) in the study presented for examination during the first 10 to 14 days of their illness with a few patients presenting as late as one month. This late presentation can be attributed to various factors including - poor transportation, poor socioeconomic condition, low literacy rate and public awareness. This is consistent with findings in Nepal, where only $43.7 \%$ took prompt medical attention within a week and 19.3\% taking as long as a month to present in the hospital. ${ }^{10}$

In our study we found that, treatment was taken by 86 $(70.5 \%)$ of the 122 patients before their presentation at Silchar Medical College and Hospital. It was interesting to 
note that a significant number of patients $(30.3 \%)$ received advice from untrained physicians. In several instances patients consulted both physicians and village healers before presenting for examination. This is similar to the findings in South India ${ }^{12}$ and Malawi. ${ }^{13}$ Of the 86 patients who presented on some form of topical medication, 51 patients (59.3\%) were taking topical antibiotics, usually moxifloxacin, ciprofloxacin, gentamicin or chloramphenicol; 8 patients (9.3\%) were taking antifungal drops, usually natamycin. It was alarming to note that 17 patients $(19.7 \%)$ were taking topical antibiotic and corticosteroids fixed dose combination eye drop; and 10 (11.6\%) were taking unknown drops. Use of chloramphenicol, gentamicin, topical corticosteroids and herbal products were seen to be in cases who visited pharmacists, homeopaths, chemists or quacks.

In our study, we found that a positive history of recent corneal trauma in $41(33.6 \%)$ of the 122 patients. In most of the patients the causative agent was agricultural or animal products, rest could not specify the causative agent. This correlated with other studies conducted.10,12,14,15 Other complicating conditions predisposing to corneal ulceration were present in $21(17.2 \%)$ of the 122 patients. These conditions included chronic dacryocystitis in 7 patients, 9 patients had uncontrolled diabetes, 2 patients presented with entropion with trichiasis and 3 patients had exposure keratitis due to Bell's palsy. Similar findings were note by Srinivasan et al.12 and Basak et al. ${ }^{16}$ However unlike international studies ${ }^{17}$ the incidence of contact lens related corneal ulcer was not seen, probably due to poor socioeconomic backgrounds of the patient. In our study, with regard to the microbial flora, out of 70 positive isolates, pure bacterial growth was present in 37 (52.8\%) cultures and pure fungal growth in 23 (32.8\%) cultures. In a total of 10 (14.3\%) mixed growth was seen. Bashir et al. showed a bacterial yield of $40 \%$ almost similar to our study and fungal growth was found in $12.5 \%$ of cases. ${ }^{18}$ The study conducted in Madurai showed pure bacterial growth in $32.3 \%$ and pure fungal growth in $32.0 \%$ of the cultures. ${ }^{12}$ However, Keshav et al. found a low fungal yield of $11.5 \%$, probably due to low incidence of ocular trauma. ${ }^{20}$

In our study, Staphylococcus aureus was the most commonly isolated $(42.5 \%)$ bacterial organism, followed by Streptococcus pneumoniae (15\%) and Pseudomonas aeruginosa (15\%). In the Madurai study, Streptococcus pneumoniae was the most commonly isolated (44.3\%). ${ }^{12}$ Keshav et al., in South Sharqiya region reported Pseudomonas to be the commonest isolate (53.84\%). ${ }^{19}$ In several other studies, Pneumococci was the most common isolate. ${ }^{10,19}$ In a study by Laspina et al, coagulase negative Staphylococcus was the most common isolate ${ }^{20}$ while Leck et al. found Pseudomonas to be the prime isolate amongst the bacterial ulcers in Ghana. ${ }^{21}$ The most common fungal isolate was Aspergillus spp. (46.7\%) in our study. In most of the world Aspergillus spp. or Candida spp. are the predominant fungal pathogens responsible for mycotic keratitis. ${ }^{22}$ This diversity has been attributed to different climatic conditions, socioeconomic standards, culture and occupation that are seen in these geographical areas.

\section{CONCLUSIONS}

Although the major aetiological agents in our study were bacterial, fungal ulcers were also seen in a significant proportion of patients. Staphylococcus was the major bacterial isolate and Aspergillus was the major fungal isolate. Monotherapy with Moxifloxacin eye drop was effective in treating a majority of corneal ulcers. Fortified Cefotaxime and Amikacin eye drops were used in cases with positive sensitivity reports. For fungal isolates, Natamycin eye drops were used.

\section{REFERENCES}

[1] The prevention of blindness: report of a WHO Study Group. Geneva, World Health Organization, WHO Technical Report Series, No. 518. 1973: p. 10-11.

[2] Smith GT, Taylor HR. Epidemiology of corneal blindness in developing countries. Refractive and Corneal Surgery 1991;7(6):436-9.

[3] Di Bisceglie AM, Carmichael TR. Factors predisposing to central corneal ulceration in a developing population. S Afr Med J 1987;71(12):769-70.

[4] Gonzales CA, Srinivasan M, Whitcher JP, et al. Incidence of corneal ulceration in Madurai District, South India. Ophthalmic Epidemiol 1996;3(3):159-66.

[5] Erie JC, Nevitt MP, Hodge DO, et al. Incidence of ulcerative keratitis in a defined population from 1950 through 1988. Arch Ophthalmol 1993;111(12):1665-71.

[6] Chirambo MC, Benezra D. Causes of blindness among students in blind school institutions in a developing country. British Journal of Ophthalmology 1976;60(9):665-8.

[7] Brilliant LB, Pokhrel RP, Grasset NC, et al. Epidemiology of blindness in Nepal. Bulletin of the World Health Organization 1985;63(2):375-86.

[8] Gilbert CE, Wood M, Waddel K, et al. Causes of childhood blindness in east Africa: results in 491 pupils attending 17 schools for the blind in Malawi, Kenya and Uganda. Ophthalmic Epidemiology 1995;2(2):77-84.

[9] Thylefors B, Megrel AD, Pararajasegaram R, et al. Available data on blindness (Update 1994). Ophthalmic Epidemiology 1995;2(1):5-39.

[10] Upadhyay MP, Karmacharya PC, Koirala S, et al. Epidemiologic characteristics, predisposing factors and etiologic diagnosis of corneal ulceration in Nepal. American Journal of Ophthalmology 1991;111(1):92-9.

[11] Hagan M, Wright E, Newman M, et al. Causes of suppurative keratitis in Ghana. British Journal of Ophthalmology 1995;79(11):1024-8.

[12] Srinivasan M, Gonzales CA, George C, et al. Epidemiology and aetiological diagnosis of corneal ulceration in Madurai, south India. British Journal of Ophthalmology 1997;81(11):965-71.

[13] Courtright P, Lewallen S, Kanjaloti S, et al. Traditional eye medicine use among patients with corneal disease in rural Malawi. British Journal of Ophthalmology 1994;78(11):810-2. 
[14] Jones DB. Decision-making in the management of microbial keratitis. Ophthalmology 1981;88(8):814-20.

[15] Jeng BH, Holsclaw DS, Kumar AB, et al. Epidemiology of corneal ulcers in Northern California. Invest Ophthalmol \& Vis Sci 2003;44(13):805.

[16] Basak SK, Basak S, Mohanta A, et al. Epidemiological and microbiological diagnosis of suppurative keratitis in Gangetic West Bengal, eastern India. Indian J Ophthalmol 2005;53(1):17-22.

[17] Keay L, Edwards K, Naduvilath T, et al. Microbial keratitis predisposing factors and morbidity. Ophthalmology 2006;113(1):109-16.

[18] Bashir G, Shah A, Thokar MA, et al. Bacterial and fungal profile of corneal ulcers-a prospective study. Indian J Pathol Microbiol 2005;48(2):273-7.
[19] Keshav BR, Zacheria G, Ideculla T, et al. Epidemiological characteristics of corneal ulcers in South Sharqiya region. Oman Med J 2008;23(1):34-9.

[20] Laspina F, Samudio M, Cibilis D, et al. Graefes Epidemiological characteristics of microbiological results on patients with infectious corneal ulcers: A 13 year survey in Paraguay. Graeles Arch Clin Exp Ophthalmology 2004;242(3):204-9.

[21] Leck AK, Thomas PA, Hagan M, et al. Aetiology of suppurative corneal ulcers in Ghana and south India, and epidemiology of fungal keratitis. British Journal of Ophthalmology 2002;86(11):1211-5.

[22] Jones BR. Principles in the management of oculomycosis. XXXI Edward Jackson Memorial Lecture. American Journal of Ophthalmology 1975;79(5):719-51. 